\title{
Experimental Comparison of Face detection Techniques on the basis of Intrinsic and Extrinsic Parameters
}

\author{
Jebran Khan \\ Department of Computer \\ Systems Engineering, \\ University of Engineering \& \\ Technology, Peshawar.
}

\author{
Nasru-minnallah \\ Department of Computer \\ Systems Engineering, \\ University of Engineering \& \\ Technology, Peshawar.
}

\author{
Hurmat Ali Shah \\ Department of Computer \\ Systems Engineering, \\ University of Engineering \& \\ Technology, Peshawar.
}

\begin{abstract}
Face detection is an important contributing factor to make computer vision applicable to problems of everyday life. Of the techniques used for face detection, two are more efficacious i.e. skin detection and violajones based face detection. These two techniques have limitations of their own. One of the most restricting factors to these techniques is the color cluster of the image. Face detection algorithms are trained on a set of images. Those images are sure to be occupying a particular color cluster of the color plane because it is not possible for the set to contain encompass the full color space. If an image occupying a color space other than that on which the algorithms is trained is an input to the algorithm, the result is not optimal then. This paper explores the effect of different color clusters on the performance of both techniques. Images of color clusters other than that on which the algorithms are trained are given to the algorithms and the results are analyzed to decide the optimality of the techniques in face of images on which the algorithms are not trained.
\end{abstract}

\section{Key-words:}

Viola-jones, Face detection, Color cluster, Color spaces.

\section{INTRODUCTION}

Skin detection make us able to find different human body parts and its applications is spread over a wide range of operations i.e. from face detection to human hand and other body parts detection; it is also useful in recognition of humans, stored in a database, in an image. The skin detection can also used be in filters which are used for blocking of objectionable contents. Skin detection is the first task in computer vision towards face detection [1]. For human faces the skin color is used as distinguishing feature. And in a simple background image the skin detection based face detection is used [2].

The face detection task makes the area of computer vision able to implement the real world problems such as biometrics security systems [3]. It is of great interest over years and there are different techniques that are used for skin detection. Different approaches for face detection are discussed in [4] [5]. The author in the review papers [4] [5] has divided the face detection techniques in the following four categories: (1) Feature invariant method is based on the principle to find structural features which do not vary with variation in the position, viewpoints or illumination conditions of an image and then use these feature in order to find face [6]. (2) Knowledge based approach aims to use rule-based approaches to encode knowledge of the about the typical facial structure. Normally the rules contain the relationship among the features of the face [7]. (3) Appearance based approaches: In this method the technique is trained on a set of training images, which contain faces of different kinds, from which models of faces are extracted. And the models are then used for detection [8]. (4) Template matching approaches: In this approach many standard facial patterns, which describe a whole face or various facial features separately, are stored. And for face detection the correlation between the stored template and the input image is computed [9].

In this paper the results of experiments done on two most important face detection techniques i.e. viola-jones and face detection based on skin detection, are shown. The effect of different color clusters on these two techniques is studied. It is shown here the impact that both these techniques are prone to color clusters of different kind.

The viola-jones face detection framework takes its decision on the basis of different features of the image. This technique is based on three features. The two rectangles feature whose value is the difference between pixel values in the two rectangular regions. The shape and size of the region are the same and they are horizontally and vertically adjacent. The three rectangle feature whose values are computed as, sum the two outside rectangles and subtract it from the center rectangle. And at last the four rectangle feature values are computed as the difference of the diagonal pairs of rectangle. These features result in a new image known as integral image [10]. These features may depend on the illumination, image orientation, image color spaces and saturation. The skin detection technique uses the pixel color to classify it as skin or not [3].

Various efforts are made to improve the skin detection techniques. (Haddi et al., 2002) developed an approach for detecting color images in different conditions this approach was developed using skin locus and hierarchal detector. (Viola, 2004) improved his viola-jones framework developed for detection in [10], and they extended it to the non frontal faces. For different views of faces different detectors were designed and a trained a decision tree for determining the image class. (Sahbi et al., 2006) used skin color and image segmentation for face detection. (Singh, 2006) carried out an experimental work on face detection based on skin color for different color spaces. 
There is a lot of other work published on the face detection. Here the focus is on comparison of the two, viola-jones framework and face detection based on skin detection, based analysis of their performance for images having different color clusters.

\section{METHODOLOGY}

\subsection{Background}

People often use skin color and textures to infer different aspects about each other. It can be used to indicate race, health, beauty, age etc. [11]. However these interpretations may vary across cultures. The existence of skin color in video or an image indicates the existence of human in that media. So, during the last few decades a lot research focused on the skin detection in an image.

Our task here is to compare the two techniques of face detection i.e. face detection using skin detection algorithm and viola-jones framework, on the basis of skin color clusters. This paper is not to describe the complexity of the two algorithms. Here we will only discuss them according to our targeted parameters. The skin detection is detecting the pixel or region that contains skin tone color. The face detection using skin detection works on the concept of color space. The region or space that is occupied by a particular class of color is known as color cluster. So the skin color of different ethnicities occupies a specific color regions and that is called the skin color cluster. And for skin detection technique the basic principle is that it uses the skin color clusters and declare it either a skin pixel or not (Eggamal et al., 2009). On the other hand the viola-jones frame work's decision is dependent on the values of simple facial features. These facial features may be space between eye-brows, the distance between the fore-head and the nose tip, the cheeks etc. These features lie in specific regions. These regions can be detected by using two rectangles features window, three rectangles features window and four rectangles features window.

\subsection{Color spaces for skin modeling}

Many color spaces took birth from the field of computer graphics and they are having different properties. And of them a wide verity has applied to modeling of skin color. The most famous of them are briefly discussed here.

\subsubsection{RGB (Red, Green, Blue)}

This color space came into being from CRT (Cathode Ray Tube) display application, when color was characterized as mixture of the three basic colored rays i.e. red, green and blue. Among all the color spaces used RGB is among the most commonly used color space for digital image data manipulating and storing. However, due to high correlation among the channels, intermingling of luminance and chrominance data and significant perceptual non-uniformity the RGB is not a favorable choice for color analysis algorithms and recognition algorithms based on color [12]. The RGB color space was used in [13] [14].

\subsubsection{Normalized $R G B$}

It is calculated from the values of the three components i.e. red, green and blue, of RGB simply by normalization method:

$$
\begin{aligned}
& r=\frac{R}{R+G+B} \\
& g=\frac{G}{R+G+B} \\
& b=\frac{B}{R+G+B}
\end{aligned}
$$

The cumulative sum of all the normalized components is $(r+g+b=1)$, and as there is no important information contained in the third component so it can be neglected. The other two components are termed as "pure colors"; RGB is reduced by normalization due to dependence of red and green on the brightness. For dull surfaces, while neglecting the environmental light, the normalized RGB do not changes with changes in the surface orientation, relative to source of light; it is a significant property of the normalized RGB [15]. This and the transformation simplicity made this color-space popular among the researchers [16] [17].

\subsubsection{HSI ( Hue Saturation Intensity)}

HSI was introduced when the user needed to stat the properties of color numerically. HSI described the color with the perceptive values that is based on the idea of an artist about hue, saturation and quality. Hue represents the principal or the dominant color (red, green, yellow, purple etc) of a region; saturation is the measurement of the colorfulness of a region relative to its intensity [18]; the intensity is associated with the luminosity of the color. HSI is popular in the skin segmentation work because of the color space component's perception and clear discrimination among the luminance and chrominance properties [17] [19]. Many remarkable features of Hue were illustrated in [15]: it does not change with highlights at the sources of white light, and same, for dull surfaces, to the environmental light and position of the surface relative to source of light. However, [18] pointed out many unwanted properties of this color space, which includes the discontinuities of the hue and the brightness computation, which conflicts with the color vision features [20].

\subsection{4. $\quad \mathrm{YCrCb}$}

It is a nonlinear and encoded RGB signal, which is commonly used for compression of image by European television studio. The color is represented by luminance calculated from nonlinear RGB [18], and two color difference values i.e. $\mathrm{Cr}$ and $\mathrm{Cb}$, the luminance is also called the luma and is represented by $Y$, luma is weighted sum of values of the components of RGB. $\mathrm{Cr}$ And $\mathrm{Cb}$ are determined by subtracting the value of luma from red and blue color components of the RGB respectively.

$$
\begin{gathered}
Y=a R+b G+c B \\
C r=R-Y \\
C b=B-Y
\end{gathered}
$$

This color space is very popular in modeling of skin color because of the simplicity of transformation and also that the components of the luminance and chrominance are explicitly separated [21] [17].

\section{PARAMETERS}

We are using the color space, position of faces in an image, illumination and complexity of an image as our comparison parameter. We are analyzing the 
performance both our face detection techniques by applying it to the images of different types. We are comparing the results of both techniques.

\section{RESULTS}

The below are the experimental results of both face detection techniques and skin tone detection technique applied on different images.

The image that contain the skin tone color in the background are considered to be one object by the skin detection base face detection technique while the violajones works good for it. Also in this image the position of the face is towards the camera and the features of the face are very much recognizable so the viola-jones detected the face.
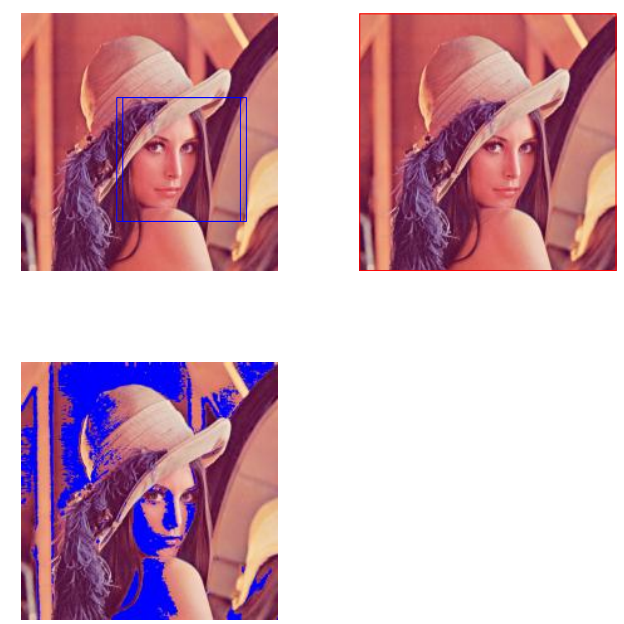

Fig1: Image containing Skin-tone color in the background.

When an image contain many faces of different color clusters then the skin color based face detection technique consider one color cluster to be the skin color and detect it as a face. The viola-jones works comparatively good in this scenario and detects many faces according to its feature selection criteria. However the viola-jones also has some false detections i.e. it declared the non face area to be the face in some places. But as a whole it only missed quite a few faces which mean the viola-jones works fine in case of complex images i.e. an image having more faces. In Fig2: all the faces are having different illumination so the skin color based face detection technique failed to detect the other face with different illumination and only detects a face in an image with having specific illumination. The skin color based face detection considered the whole place in the image to be a face, where the density of the skin tone color is high and the regions are connected as in the Fig3: Also the viola-jones detected the faces of different colors in a single image.
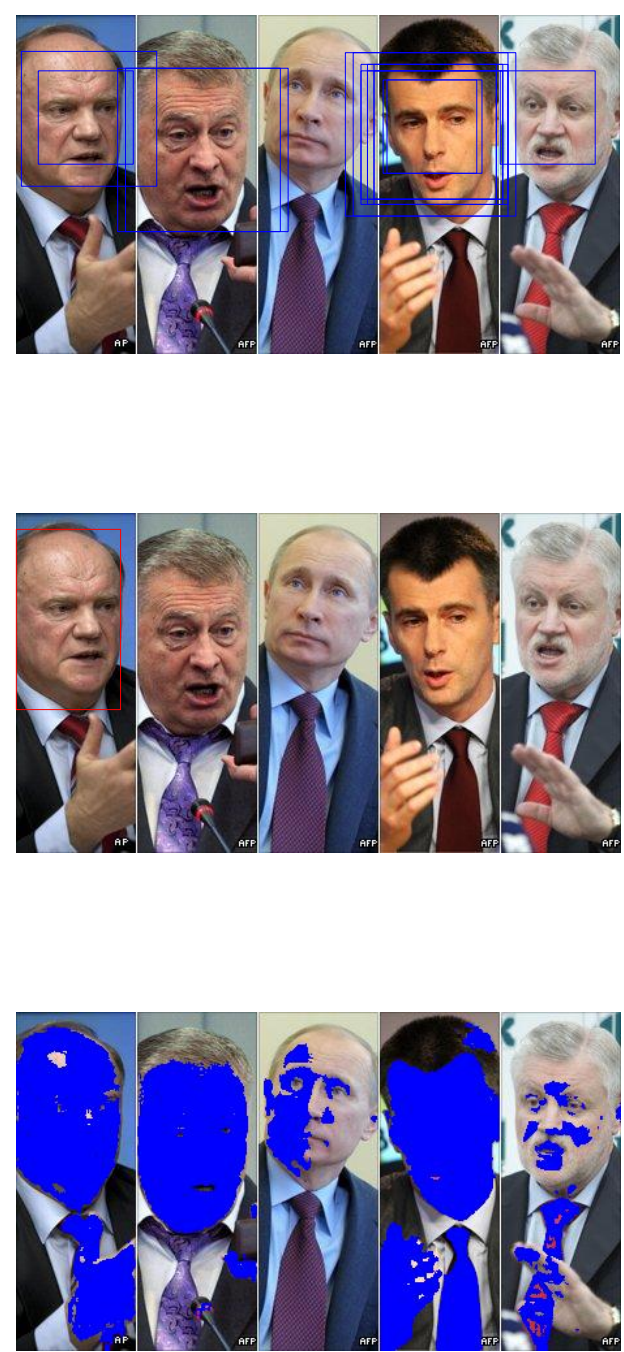

Fig2: Image having multiple faces of different color clusters
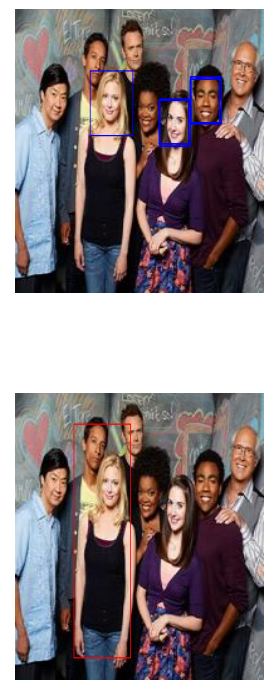

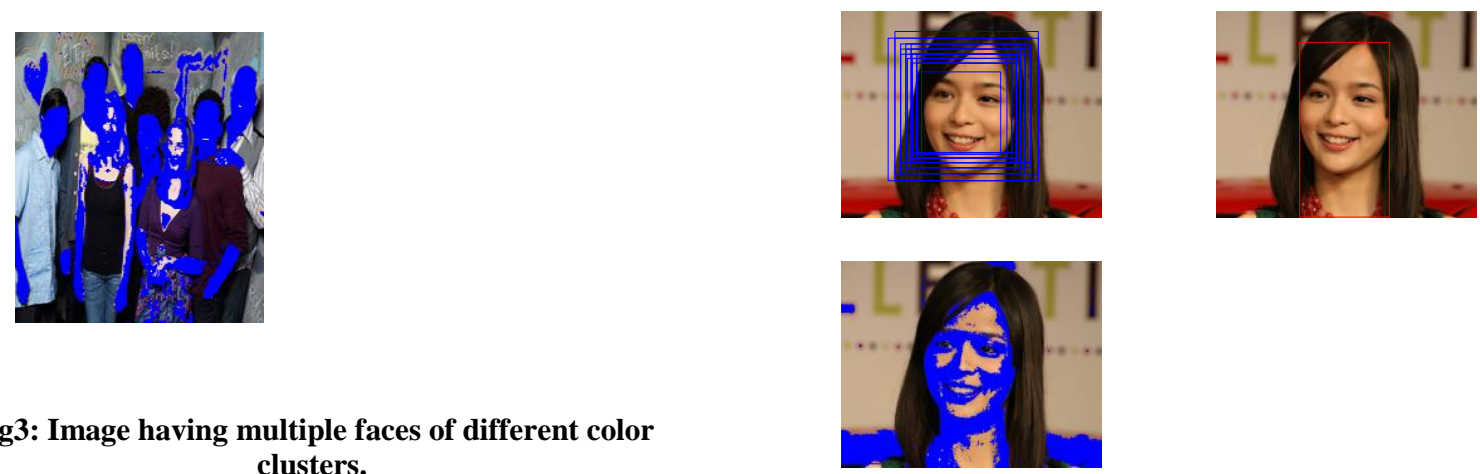

Fig3: Image having multiple faces of different color clusters.

The skin detection based face detection in some cases do false detection when the density of red color, as compared to face is higher in the background. The violajones works good and detected the face.
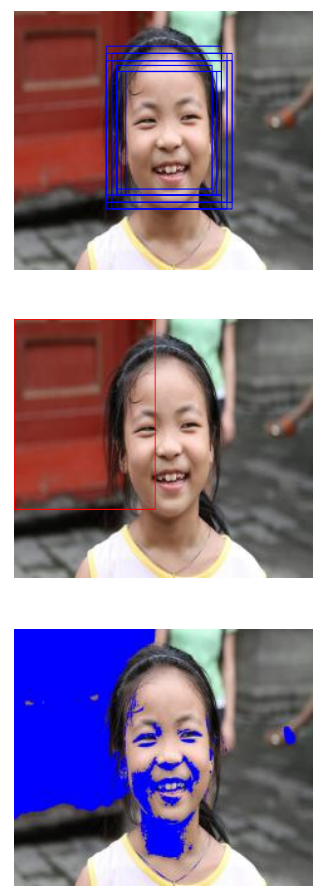

Fig4: Image containing more red color density in the background.

Here in Fig5; the image background contain red color but its density is low so the face is detected correctly by the skin color based face detection but there is also some false detection i.e. neck region is also considered as face.
Fig5: Image containing low red color density in the background.

In Fig6: the skin color based face detection is working good as it is more likely to the above image but here the viola-jones failed because in this image one eye is not clearly visible and as viola-jones detects face on the basis of features so it failed classify it as a face. Here the position of the face is towards the camera but there are some hairs on one eye and therefore the viola-jones did not recognize it to be the face. While the skin color based face detection technique worked the same as was in the Fig5.
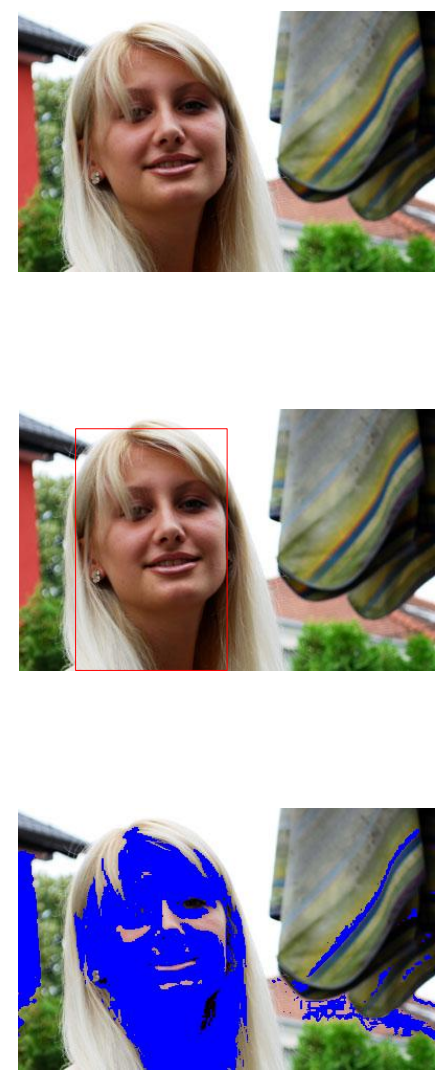

Fig6: Image in which all the features are not clearly visible. 
The same is the case with image in Fig7: the features are not recognized to be that of the face. As it is an avatar image so the face feature of it is different from the humans for whom this algorithm is trained and so not detected by the viola-jones. Here the skin color based face detection technique done a false detection as the color is different from the skin color for which the algorithm is trained. In this image both the face detection techniques failed to detect faces.
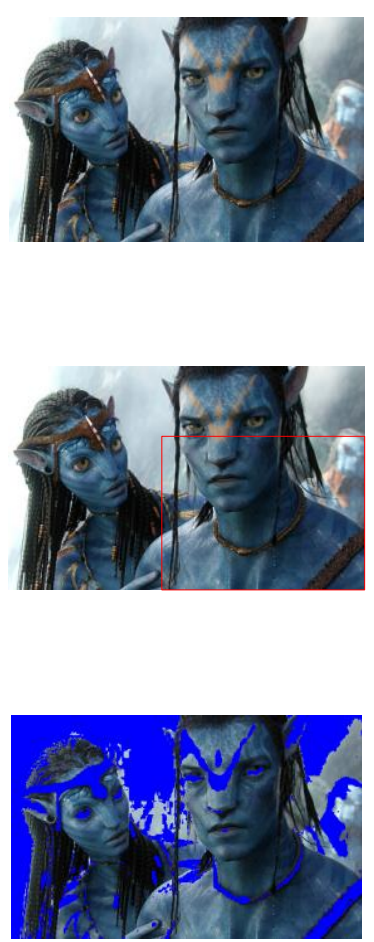

Fig7: Avatar image

A small patch in the image was identified as skin color, so one face that contains that patch was declared as face in Fig8. While viola-jones was able to detect those faces that has its features more prominent. Here the position of some faces is different and in those images where the features of the face are more visible are detected as face by viola-jones.

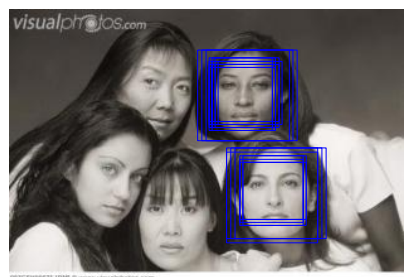

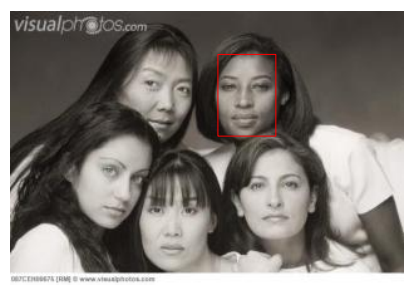

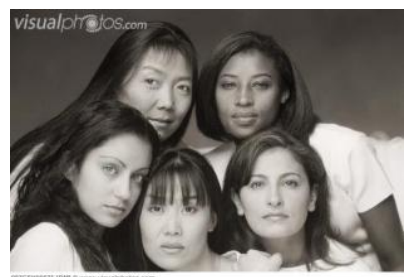

Fig8: Black and White image

\section{CONCLUSION}

The results show the inefficacy of skin detection based face detection when the image color space is composed of many color clusters. False positive rate is maximized in such cases. Another shortcoming of the skin detection based face detection is the dependency on which the algorithm has been trained. The results contain the skin patches contain in the image. The algorithm was trained on restricted varieties of skin color, so despite that the image has many skin-tone colors, the algorithm fails to detect the faces that belong to color clusters on which the algorithms has not been trained. Often many skin-tone color regions, though disperse, are combined in a false way to declare a false face. The exposed parts of human body that have skin-tone colors belonging to the algorithm color space are declared as face, while the original face that have skin-color lying outside the algorithm trained color space is not declared as face. Skin detection also misled to declaring a whole region containing many faces as one single face when that region is composed of skin-tone colors on which the algorithm has been trained. Viola-Jones does optimally in all of the discussed scenarios. Its harrrs-cascade feature extraction make it immune to the variation in color space or the multiplicity of color clusters in the color space of an image. So in case of multi-colored image scenario and complex images, viola-jones outperforms the skin-based techniques. The skin detection based face detection do not depends on the position of the face in an image and clarity of the facial features, so in those scenarios where the face position is not clear the skin color based technique is more useful to be used. The efficiency of the viola-jones is not affected by the illumination and complexity of the image, so in more complex environment and for images with different illumination levels the viola-jones is the more appropriate technique to be used. Also the skin detection is very prone to false detection and therefore in those cases where the false detection can harm the ultimate goal then the viola-jones is the best option while in cases where the false detection is acceptable then the skin color based technique is fine to be used. The viola-jones is complex and also can take more execution time while the skin color based technique is relatively simple so in case 
where the false detection is not a problem and where a controlled environment can be provided the skin color based technique can be a good option e.g. database searching. While in case of real life problems where the false detection can create problems the viola-jones is better to be used. In some cases where one may be interested in detecting some other object along with face in an image such as in nude images.

\section{REFERENCES}

[1] Rehanullah Khan, Allan Hanbury, Julian St $\square$ ottinger, Abdul Bais,: Color Based Skin Classification. CVL, Institute of Computer Aided Automation, TU-Wien, Austria.

[2] Ahmed Elgammal, Crystal Muang and Dunxu Hu: Skin Detection - a Short Tutorial. Department of Computer Science, Rutgers University, Piscataway, NJ, 08902, USA.

[3] H. A. SHAH, R. KHAN, L. HASAN, J. KHAN, A. KHTTAK, S. JAN, "On Experimental Comparison of Color and Supervised Face Detection" , Sindh Univ. Res. Jour. (Sci. Ser.) Vol.44 (4) 561-564 (2012).

[4] Ming-Hsuan Yang, David J. K, Narenda. A, "Detecting Faces in Images: A Survey", IEEE Trans. On Pattern Analysis \& Machine Intelligence, vol. 24, no.1, January 2002.

[5] Erik Hjelmas, Boon K. L, "Face Detection: A Survey", Computer Vision and Image Understanding, 83, pg. 236-274, 2001.

[6] K.C. Yow and R. Cipolla, "Feature-Based Human Face Detection," Image and Vision Computing, vol. 15, no. 9, pp. 713-735, 1997.

[7] G. Yang and T. S. Huang, "Human Face Detection in Complex Background," Pattern Recognition, vol. 27, no. 1, pp. 53-63, 1994.

[8] K.-K. Sung and T. Poggio, "Example-Based Learning for View-Based Human Face Detection," IEEE Trans. Pattern Analysis and Machine Intelligence, vol. 20, no. 1, pp. 39-51, Jan. 1998.

[9] I. Craw, D. Tock, and A. Bennett, "Finding Face Features," Proc. Second European Conf. Computer Vision, pp. 92-96, 1992.

[10] PAUL VIOLA, MICHAEL J. JONES,: Robust Real-Time Face Detection. Received September 10, 2001; Revised July 10, 2003; Accepted July 11, 2003.
[11] Bernhard Fink, K.G., Matts, P.J.: Visible skin color distribution plays a role in the perception of age, attractiveness, and health in female faces. Evolution and Human Behavior 27(6) (2006) 433-442.

[12] Vladimir Vezhnevets, Vassili Sazonov, Alla Andreeva. A Survey on Pixel-Based Skin Color Detection Techniques. International Conference Graphicon 2003, Moscow, Russia, http://www.graphicon.ru/.

[13] BRAND, J., AND MASON, J. 2000. A comparative assessment of three approaches to pixellevel human skin-detection. In Proc. of the International Conference on Pattern Recognition, vol. 1, 10561059.

[14] JONES, M. J., AND REHG, J. M. 1999. Statistical color models with application to skin detection. In Proc. of the CVPR '99, vol. 1, 274-280.

[15] SKARBEK, W., AND KOSCHAN, A. 1994. Colour image segmentation - a survey -. Tech. rep., Institute for Technical Informatics, Technical University of Berlin, October.

[16] BROWN, D., CRAW, I., AND LEWTHWAITE, J. 2001. A som based approach to skin detection with application in real time systems. In Proc. of the British Machine Vision Conference, 2001.

[17] ZARIT, B. D., SUPER, B. J., AND QUEK, F. K. H. 1999. Comparison of five color models in skin pixel classification. In ICCV'99 Int'l Workshop on recognition, analysis and tracking of faces and gestures in Real-Time systems, 58-63.

[18] POYNTON, C. A. 1995. Frequently asked questions about color. In ftp://www.inforamp.net/pub/users/poynton/doc/colo ur/ColorFAQ.ps.gz.

[19] MCKENNA, S., GONG, S., AND RAJA, Y. 1998. Modeling facial color and identity with gaussian mixtures. Pattern Recognition 31, 12, 1883- 1892.

[20] Macro Anisetti, Fast and Robust Face Detection, Multimedia Techniques for Device and Ambient Intelligence, Springer US, 2009, pp 43-72.

[21] PHUNG, S. L., BOUZERDOUM, A., AND CHAI, D. 2002. A novel skin color model in ycbcr color space and its application to human face detection. In IEEE International Conference on Image Processing (ICIP'2002), vol. 1, 289-292. 